\title{
Matrix Inequalities for the Fan Product and the Hadamard Product of Matrices
}

\author{
Dongjie Gao \\ Department of Mathematics, Heze University, Heze, China \\ Email: aizai_2004@126.com
}

Received 6 July 2015; accepted 29 August 2015; published 1 September 2015

Copyright (C) 2015 by author and Scientific Research Publishing Inc.

This work is licensed under the Creative Commons Attribution International License (CC BY). http://creativecommons.org/licenses/by/4.0/

(c) (i) Open Access

\begin{abstract}
A new inequality on the minimum eigenvalue for the Fan product of nonsingular $M$-matrices is given. In addition, a new inequality on the spectral radius of the Hadamard product of nonnegative matrices is also obtained. These inequalities can improve considerably some previous results.
\end{abstract}

\section{Keywords}

\section{M-Matrix, Nonnegative Matrix, Fan Product, Hadamard Product, Spectral Radius, Minimum} Eigenvalue

\section{Introduction}

Let $A=\left(a_{i j}\right) \in \mathbb{R}^{n \times n}$, and $N=\{1,2, \cdots, n\}$. We write $A \geq 0 \quad(A>0)$ if $a_{i j} \geq 0 \quad\left(a_{i j}>0\right)$ for any $i, j \in N$. If $A \geq 0, A$ is called a nonnegative matrix, and if $A>0, A$ is called a positive matrix. The spectral radius of a nonnegative matrix $A$ is denoted by $\rho(A)$.

We denote by $Z_{n}$ the class of all $n \times n$ real matrices, all of whose off-diagonal entries are nonpositive. A matrix $A=\left(a_{i j}\right) \in Z_{n}$ is called an $M$-matrix if there exists a nonnegative matrix $B$ and a nonnegative real number $s$, such that $A=s I-B$ with $s \geq \rho(B)$, where $I$ is the identity matrix. If $s>\rho(B)$ (resp., $s=\rho(B))$, then the $M$-matrix $A$ is nonsingular (resp., singular) (see [1] [2]). Denote by $M_{n}$ the set of nonsingular $M$-matrices. We define $\tau(A)=\min \{\operatorname{Re}(\lambda): \lambda \in \sigma(A)\}$, where $\sigma(A)$ denotes the spectrum of $A$.

The Fan product of two matrices $A=\left(a_{i j}\right) \in \mathbb{C}^{n \times n}$ and $B=\left(b_{i j}\right) \in \mathbb{C}^{n \times n}$ is the matrix $A \star B=\left(c_{i j}\right) \in \mathbb{C}^{n \times n}$, where

$$
c_{i j}= \begin{cases}-a_{i j} b_{i j}, & \text { if } i \neq j, \\ a_{i i} b_{i i}, & \text { if } i=j .\end{cases}
$$

If $A, B \in M_{n}$, then so is $A \star B$. In ([2], p. 359), a lower bound for $\tau(A \star B)$ was given: if $A, B \in M_{n}$, 
then $\tau(A \star B) \geq \tau(A) \tau(B)$.

If $A=\left(a_{i j}\right) \in M_{n}$, and $a_{i i}>0$, we write $Q=D-A$, where $D=\operatorname{diag}\left(a_{i i}\right)$. Thus we define $J_{A}=D^{-1} Q$. Obviously, $J_{A}$ is nonnegative. Recently, some authors gave some lower bounds of $\tau(A \star B)$ (see [3]-[8]). In [4], Huang obtained the following result for $\tau(A \star B)$,

$$
\tau(A \star B) \geq\left(1-\rho\left(J_{A}\right) \rho\left(J_{B}\right)\right) \min _{1 \leq i \leq n}\left\{a_{i i} b_{i i}\right\} .
$$

The bound of (1) is better than the bound $\tau(A) \tau(B)$ in ([2], p. 359).

In [7], Liu gave a lower bound of $\tau(A \star B)$,

$$
\tau(A \star B) \geq \min _{i \neq j} \frac{1}{2}\left\{a_{i i} b_{i i}+a_{j j} b_{j j}-\left[\left(a_{i i} b_{i i}-a_{j j} b_{j j}\right)^{2}+4 \Gamma_{i j}\right]^{\frac{1}{2}}\right\},
$$

where $\Gamma_{i j}=a_{i i} a_{i j} b_{i i} b_{j j} \rho^{2}\left(J_{A}\right) \rho^{2}\left(J_{B}\right)$. The bound of (2) is better than the one of (1).

For a nonnegative matrix $A=\left(a_{i j}\right)$, let $N=A-D$, where $D=\operatorname{diag}\left(a_{i i}\right)$. We denote $J_{A}^{\prime}=D_{1}^{-1} N$, where $D_{1}=\operatorname{diag}\left(d_{i i}\right)$,

$$
d_{i i}= \begin{cases}a_{i i}, & \text { if } a_{i i} \neq 0, \\ 1, & \text { if } a_{i i}=0 .\end{cases}
$$

The Hadamard product of two matrices $A=\left(a_{i j}\right) \in \mathbb{C}^{n \times n}$ and $B=\left(b_{i j}\right) \in \mathbb{C}^{n \times n}$ is the matrix $A \circ B=\left(a_{i j} b_{i j}\right) \in \mathbb{C}^{n \times n}$. For two nonnegative matrices $A$ and $B$, recently, some authors gave several new upper bounds of $\rho(A \circ B)$ (see [3]-[7] [9]). In [4], Huang obtained the following result for $\rho(A \circ B)$,

1) If $a_{i i} b_{i i} \neq 0, \forall i \in N$, then

$$
\rho(A \circ B) \leq\left(1+\rho\left(J_{A}^{\prime}\right) \rho\left(J_{B}^{\prime}\right)\right) \max _{1 \leq i \leq n}\left\{a_{i i} b_{i i}\right\} .
$$

2) If $a_{i_{0} i_{0}} \neq 0$ or $b_{i_{0} i_{0}} \neq 0$ for some $i_{0}$, but $a_{i i} b_{i i}=0, \forall i \in N$, then

$$
\rho(A \circ B) \leq \rho\left(J_{A}^{\prime}\right) \rho\left(J_{B}^{\prime}\right) \max _{1 \leq i \leq n}\left\{a_{i i}, b_{i i}\right\} .
$$

3) If $a_{i i}=0$ and $b_{i i}=0, \forall i \in N$, then

$$
\rho(A \circ B) \leq \rho\left(J_{A}^{\prime}\right) \rho\left(J_{B}^{\prime}\right) .
$$

4) If $a_{i_{0} i_{0}} b_{i_{0} i_{0}} \neq 0$ and $a_{j_{0} j_{0}} b_{j_{0} j_{0}} \neq 0$ for some $i_{0}, j_{0}$, then the upper bound of $\rho(A \circ B)$ is the maximum value of the upper bounds of the inequalities in (3)-(5).

The bound of $\rho(A \circ B)$ in [4] is better than that in ([2], p. 358).

In [7], Liu gave a new upper bound of $\rho(A \circ B)$,

1) If $a_{i i} b_{i i} \neq 0, \forall i \in N$, then

$$
\rho(A \circ B) \leq \max _{i \neq j} \frac{1}{2}\left\{a_{i i} b_{i i}+a_{i j} b_{j j}+\left[\left(a_{i i} b_{i i}-a_{i j} b_{j j}\right)^{2}+4 \Omega_{i j}\right]^{\frac{1}{2}}\right\},
$$

where $\Omega_{i j}=a_{i i} a_{i j} b_{i i} b_{i j} \rho^{2}\left(J_{A}^{\prime}\right) \rho^{2}\left(J_{B}^{\prime}\right)$.

2) If $a_{i_{0} i_{0}} \neq 0$ and $a_{j_{0} j_{0}} \neq 0$ or $b_{i_{0} i_{0}} \neq 0$ and $b_{j_{0} j_{0}} \neq 0$ for some $i_{0}, j_{0}$, but $a_{i i} b_{i i}=0, \forall i \in N$, then

$$
\rho(A \circ B) \leq \rho\left(J_{A}^{\prime}\right) \rho\left(J_{B}^{\prime}\right) \max _{i \neq j}\left\{\left(a_{i i} a_{i j}\right)^{\frac{1}{2}},\left(b_{i i} b_{j j}\right)^{\frac{1}{2}}\right\} .
$$

3) If $a_{i i}=0$ and $b_{i i}=0, \forall i \in N$, then

$$
\rho(A \circ B) \leq \rho\left(J_{A}^{\prime}\right) \rho\left(J_{B}^{\prime}\right)
$$


4) If $a_{i_{0} i_{0}} b_{i_{0} i_{0}} \neq 0$ and $a_{j_{0} j_{0}} b_{j_{0} j_{0}} \neq 0$ for some $i_{0}, j_{0}$, then the upper bound of $\rho(A \circ B)$ is the maximum value of the upper bounds of the inequalities in (6)-(8).

The bound of $\rho(A \circ B)$ in [7] is better than that in [4].

The paper is organized as follows. In Section 2, we give a new lower bound of $\tau(A \star B)$. In Section 3 , we present a new upper bound of $\rho(A \circ B)$.

\section{Inequalities for the Fan Product of Two $M$-Matrices}

In this section, we will give a new lower bound of $\tau(A \star B)$.

If $A=\left(a_{i j}\right) \in \mathbb{R}^{n \times n}$ and $k \geq 0$, we write $A^{(k)}=\left(a_{i j}^{k}\right)$ for the $k$-th Hadamard power of $A$. If $x=\left(x_{i}\right) \in \mathbb{R}^{n}$ and $k \geq 0$, we write $x^{(k)}=\left(x_{i}^{k}\right)$.

Lemma 1. [7] Let $A, B \in M_{n}$, and let $D, E \in \mathbb{R}^{n \times n}$ be two positive diagonal matrices. Then

$$
D(A \star B) E=(D A E) \star B=(D A) \star(B E)=(A E) \star(D B)=A \star(D B E) .
$$

Lemma 2. [2] If $A=\left(a_{i j}\right) \in \mathbb{R}^{n \times n}$ is a nonnegative matrix and $k \geq 1$, then

$$
\rho\left(A^{(k)}\right) \leq \rho^{k}(A)
$$

Theorem 1. Let $A=\left(a_{i j}\right)$ and $B=\left(b_{i j}\right) \in M_{n}$. Then

$$
\tau(A \star B) \geq \min _{i \neq j} \frac{1}{2}\left\{a_{i i} b_{i i}+a_{j j} b_{j j}-\left[\left(a_{i i} b_{i i}-a_{j j} b_{j j}\right)^{2}+4 \gamma_{i j}^{(2)}\right]^{\frac{1}{2}}\right\},
$$

where $\gamma_{i j}^{(2)}=a_{i i} a_{j j} b_{i i} b_{j j} \rho\left(J_{A}^{(2)}\right) \rho\left(J_{B}^{(2)}\right), i, j \in N$.

It is evident that the Theorem holds with equality for $n=1$. Next, we assume that $n \geq 2$.

(1) First, we assume that $A \star B$ is irreducible matrix, then $A$ and $B$ are irreducible. Obviously $J_{A}$ and $J_{B}$ are also irreducible and nonnegative, so $J_{A}^{(2)}$ and $J_{B}^{(2)}$ are nonnegative irreducible matrices. Then there exist two positive vectors $\bar{u}=\left(\bar{u}_{i}\right)$ and $\bar{v}=\left(\bar{v}_{i}\right)$ such that $J_{A}^{(2)} \bar{u}=\rho\left(J_{A}^{(2)}\right) \bar{u}$ and $J_{B}^{(2)} \bar{v}=\rho\left(J_{B}^{(2)}\right) \bar{v}$. Let

$$
u=\left(u_{i}\right)=\bar{u}^{\left(\frac{1}{2}\right)}=\left(\bar{u}_{i}^{\frac{1}{2}}\right), \quad v=\left(v_{i}\right)=\bar{v}^{\left(\frac{1}{2}\right)}=\left(\bar{v}_{i}^{\frac{1}{2}}\right) \text {. }
$$

Then we have $J_{A}^{(2)} u^{(2)}=\rho\left(J_{A}^{(2)}\right) u^{(2)}$ and $J_{B}^{(2)} v^{(2)}=\rho\left(J_{B}^{(2)}\right) v^{(2)}$, that is

$$
\sum_{j \neq i} \frac{\left|a_{i j}\right|^{2} u_{j}^{2}}{u_{i}^{2}}=a_{i i}^{2} \rho\left(J_{A}^{(2)}\right), \quad \sum_{j \neq i} \frac{\left|b_{i j}\right|^{2} v_{j}^{2}}{v_{i}^{2}}=b_{i i}^{2} \rho\left(J_{B}^{(2)}\right), \quad i \in N .
$$

Let $A=\left(\tilde{a}_{i j}\right)=U^{-1} A U$ and $\tilde{B}=\left(\tilde{b}_{i j}\right)=V^{-1} B V$ in which $U$ and $V$ are the nonsingular diagonal matrices $U=\operatorname{diag}\left(u_{1}, u_{2}, \cdots, u_{n}\right)$ and $V=\operatorname{diag}\left(v_{1}, v_{2}, \cdots, v_{n}\right)$. Then, we have

$$
\tilde{A}=\left(\tilde{a}_{i j}\right)=U^{-1} A U=\left(\begin{array}{cccc}
a_{11} & \frac{a_{12} u_{2}}{u_{1}} & \ldots & \frac{a_{1 n} u_{n}}{u_{1}} \\
\frac{a_{21} u_{1}}{u_{2}} & a_{22} & \ldots & \frac{a_{2 n} u_{n}}{u_{2}} \\
\vdots & \vdots & \ddots & \vdots \\
\frac{a_{n 1} u_{1}}{u_{n}} & \frac{a_{n 2} u_{2}}{u_{n}} & \ldots & a_{n n}
\end{array}\right),
$$




$$
\tilde{B}=\left(\tilde{b}_{i j}\right)=V^{-1} B V=\left(\begin{array}{cccc}
b_{11} & \frac{b_{12} v_{2}}{v_{1}} & \cdots & \frac{b_{1 n} v_{n}}{v_{1}} \\
\frac{b_{21} v_{1}}{v_{2}} & b_{22} & \cdots & \frac{b_{2 n} v_{n}}{v_{2}} \\
\vdots & \vdots & \ddots & \vdots \\
\frac{b_{n 1} v_{1}}{v_{n}} & \frac{b_{n 2} v_{2}}{v_{n}} & \cdots & b_{n n}
\end{array}\right) .
$$

It is easy to see that $\tilde{A}, \tilde{B}$, and $V U$ are nonsingular since $V$ and $U$ are. From Lemma 1, we have

$$
(V U)^{-1}(A \star B)(V U)=U^{-1} V^{-1}(A \star B) V U=\left(U^{-1} A U\right) \star\left(V^{-1} B V\right)=\tilde{A} \star \tilde{B} .
$$

Thus, we obtain $\tau(A \star B)=\tau(\tilde{A} \star \tilde{B})$, and

$$
\tilde{A} \star \tilde{B}=\left(c_{i j}\right)=\left(\begin{array}{cccc}
a_{11} b_{11} & -\frac{a_{12} b_{12} u_{2} v_{2}}{u_{1} v_{1}} & \cdots & -\frac{a_{1 n} b_{1 n} u_{n} v_{n}}{u_{1} v_{1}} \\
-\frac{a_{21} b_{21} u_{1} v_{1}}{u_{2} v_{2}} & a_{22} b_{22} & \cdots & -\frac{a_{2 n} b_{2 n} u_{n} v_{n}}{u_{2} v_{2}} \\
\vdots & \vdots & \ddots & \vdots \\
-\frac{a_{n 1} b_{n 1} u_{1} v_{1}}{u_{n} v_{n}} & -\frac{a_{n 2} b_{n 2} u_{2} v_{2}}{u_{n} v_{n}} & \cdots & a_{n n} b_{n n}
\end{array}\right) .
$$

We next consider the minimum eigenvalue $\tau(\tilde{A} \star \tilde{B})$ of $\tilde{A} \star \tilde{B}$. Let $\lambda=\tau(\tilde{A} \star \tilde{B})$. Then we have that $0<\lambda<a_{i i} b_{i i}, \forall i \in N$. By Theorem 1.23 of [10], there exist $i_{0}, j_{0} \in N, i_{0} \neq j_{0}$, such that

$$
\left|\lambda-a_{i_{0} i_{0}} b_{i_{0} i_{0}}\right|\left|\lambda-a_{j_{0} j_{0}} b_{j_{0} j_{0}}\right| \leq \sum_{t \neq i_{0}}\left|c_{i_{0} t}\right| \sum_{t \neq j_{0}}\left|c_{j_{0} t}\right| .
$$

By Hölder's inequality, we have

$$
\begin{aligned}
\sum_{t \neq i_{0}}\left|c_{i_{0} t}\right| \sum_{t \neq j_{0}}\left|c_{j_{0} t}\right| & =\sum_{t \neq i_{0}}\left|\frac{a_{i_{0} t} b_{i_{0}} t}{u_{i_{0}} v_{i_{0}} v_{t}}\right| \sum_{t \neq j_{0}}\left|\frac{a_{j_{0} t} b_{j_{0} t} u_{t} v_{t}}{u_{j_{0}} v_{j_{0}}}\right| \\
& \leq\left(\sum_{t \neq i_{0}} \frac{\left|a_{i_{0} t}\right|^{2} u_{t}^{2}}{u_{i_{0}}^{2}} \sum_{t \neq i_{0}} \frac{\left|b_{i_{0} t}\right|^{2} v_{t}^{2}}{v_{i_{0}}^{2}} \sum_{t \neq j_{0}} \frac{\left|a_{j_{0} t}\right|^{2} u_{t}^{2}}{u_{j_{0}}^{2}} \sum_{t \neq j_{0}} \frac{\left|b_{j_{0} t}\right|^{2} v_{t}^{2}}{v_{j_{0}}^{2}}\right)^{\frac{1}{2}} \\
& =\left(a_{i_{i_{0}} i_{0}}^{2} a_{j_{0} j_{0}}^{2} b_{i_{0} i_{0}}^{2} b_{j_{0} j_{0}}^{2} \rho^{2}\left(J_{A}^{(2)}\right) \rho^{2}\left(J_{B}^{(2)}\right)\right)^{\frac{1}{2}}=\gamma_{i_{0} j_{0}}^{(2)} .
\end{aligned}
$$

Then, we have

$$
\left|\lambda-a_{i_{0} i_{0}} b_{i_{0} i_{0}}\right|\left|\lambda-a_{j_{0} j_{0}} b_{j_{0} j_{0}}\right| \leq \gamma_{i_{0} j_{0}}^{(2)} .
$$

Since $0<\lambda<a_{i i} b_{i i}, \forall i \in N$, then

$$
\left(\lambda-a_{i_{0} i_{0}} b_{i_{0} i_{0}}\right)\left(\lambda-a_{j_{0} j_{0}} b_{j_{0} j_{0}}\right) \leq \gamma_{i_{0} j_{0}}^{(2)} .
$$

Hence,

$$
\lambda \geq \frac{1}{2}\left\{a_{i_{0} i_{0}} b_{i_{0} i_{0}}+a_{j_{0} j_{0}} b_{j_{0} j_{0}}-\left[\left(a_{i_{0} i_{0}} b_{i_{0} i_{0}}-a_{j_{0} j_{0}} b_{j_{0} j_{0}}\right)^{2}+4 \gamma_{i_{0} j_{0}}^{(2)}\right]^{\frac{1}{2}}\right\},
$$

i.e., 


$$
\begin{aligned}
\tau(A \star B) & \geq \frac{1}{2}\left\{a_{i_{0} i_{0}} b_{i_{0} i_{0}}+a_{j_{0} j_{0}} b_{j_{0} j_{0}}-\left[\left(a_{i_{0} i_{0}} b_{i_{0} i_{0}}-a_{j_{0} j_{0}} b_{j_{0} j_{0}}\right)^{2}+4 \gamma_{i_{0} j_{0}}^{(2)}\right]^{\frac{1}{2}}\right\} \\
& \geq \min _{i \neq j} \frac{1}{2}\left\{a_{i i} b_{i i}+a_{j j} b_{j j}-\left[\left(a_{i i} b_{i i}-a_{j j} b_{j j}\right)^{2}+4 \gamma_{i j}^{(2)}\right]^{\frac{1}{2}}\right\} .
\end{aligned}
$$

(2) Now, assume that $A \star B$ is reducible. It is well known that a matrix in $Z_{n}$ is a nonsingular $M$-matrix if and only if all its leading principal minors are positive (see [11]). If we denote by $T=\left(t_{i j}\right)$ the $n \times n$ permutation matrix with $t_{12}=t_{23}=\cdots=t_{n-1, n}=t_{n 1}=1$, the remaining $t_{i j}$ zero, then both $A-\epsilon T$ and $B-\epsilon T$ are irreducible nonsingular $M$-matrix for any chosen positive real number $\epsilon$, sufficiently small such that all the leading principal minors of both $A-\epsilon T$ and $B-\epsilon T$ are positive. Now, we substitute $A-\epsilon T$ and $B-\epsilon T$ for $A$ and $B$, respectively, in the previous case, and then letting $\epsilon \rightarrow 0$, the result follows by continuity.

Remark 1. By Lemma 2, the bound in Theorem 1 is better than that in Theorem 4 of [8] and Theorem 2 of [7]. Example 1. Let

$$
A=\left(\begin{array}{cccc}
4 & -1 & -1 & -1 \\
-2 & 5 & -1 & -1 \\
0 & -2 & 4 & -1 \\
-1 & -1 & -1 & 4
\end{array}\right), \quad B=\left(\begin{array}{cccc}
1 & -0.5 & 0 & 0 \\
-0.5 & 1 & -0.5 & 0 \\
0 & -0.5 & 1 & -0.5 \\
0 & 0 & -0.5 & 1
\end{array}\right) .
$$

By calculating with Matlab 7.1, it is easy to show that $\tau(A \star B)=3.2296$.

Applying Theorem 4 of [4], Theorem 3.1 of [5], Theorem 2 of [7], and Theorem 3.1 of [8], we have $\tau(A \star B) \geq 1.5239, \tau(A \star B) \geq 2.4333, \tau(A \star B) \geq 1.5239$, and $\tau(A \star B) \geq 2.9779$, respectively. But, if we apply Theorem 1, we have

$$
\tau(A \star B) \geq \min _{i \neq j} \frac{1}{2}\left\{a_{i i} b_{i i}+a_{i j} b_{j j}-\left[\left(a_{i i} b_{i i}-a_{i j} b_{j j}\right)^{2}+4 \gamma_{i j}^{(2)}\right]^{\frac{1}{2}}\right\}=2.9833 .
$$

The numerical example shows that the bound in Theorem 1 is better than that in Theorem 4 of [4], Theorem 3.1 of [5], Theorem 2 of [7], and Theorem 3.1 of [8].

\section{Inequalities for the Hadamard Product of Two Nonnegative Matrices}

In this section, we will give a new upper bound of $\rho(A \circ B)$ for nonnegative matrices $A$ and $B$. Similar to [7], for $A=\left(a_{i j}\right) \geq 0$, write $Q=A-D$, where $D=\operatorname{diag}\left(a_{i i}\right)$. We denote $J_{A}^{\prime}=D_{1}^{-1} Q$ with $D_{1}=\operatorname{diag}\left(d_{i i}\right)$, where

$$
d_{i i}= \begin{cases}a_{i i}, & \text { if } a_{i i} \neq 0, \\ 1, & \text { if } a_{i i}=0 .\end{cases}
$$

Note that $J_{A}^{\prime}$ is nonnegative, and $J_{A}^{\prime}=A$ if $a_{i i}=0, \forall i \in N$. For $B=\left(b_{i j}\right) \geq 0$, let $D_{2}=\operatorname{diag}\left(\delta_{i i}\right)$, where

$$
\delta_{i i}= \begin{cases}b_{i i}, & \text { if } b_{i i} \neq 0, \\ 1, & \text { if } b_{i i}=0 .\end{cases}
$$

Similarly, the nonnegative matrix $J_{B}^{\prime}$ is defined.

Lemma 3. [2] Let $A, B \in \mathbb{R}^{n \times n}$, and let $D, E \in \mathbb{R}^{n \times n}$ be diagonal matrices. Then

$$
D(A \circ B) E=(D A E) \circ B=(D A) \circ(B E)=(A E) \circ(D B)=A \circ(D B E) .
$$

Lemma 4. [12] Let $A=\left(a_{i j}\right) \in \mathbb{R}^{n \times n}$ be a nonnegative matrix. Then

$$
\rho(A) \leq \max _{i \neq j} \frac{1}{2}\left\{a_{i i}+a_{j j}+\left[\left(a_{i i}-a_{j j}\right)^{2}+4 \sum_{k \neq i} a_{i k} \sum_{k \neq j} a_{j k}\right]^{\frac{1}{2}}\right\} .
$$


Theorem 2. Let $A=\left(a_{i j}\right), B=\left(b_{i j}\right) \in \mathbb{R}^{n \times n}, A \geq 0$ and $B \geq 0$. Then

1) If $a_{i i} b_{i i} \neq 0, \forall i \in N$, then

$$
\rho(A \circ B) \leq \max _{i \neq j} \frac{1}{2}\left\{a_{i i} b_{i i}+a_{i j} b_{j j}+\left[\left(a_{i i} b_{i i}-a_{i j} b_{j j}\right)^{2}+4 \eta_{i j}^{(2)}\right]^{\frac{1}{2}}\right\},
$$

where $\eta_{i j}^{(2)}=a_{i i} a_{i j} b_{i i} b_{j j} \rho\left(J_{A}^{\prime(2)}\right) \rho\left(J_{B}^{\prime(2)}\right), i, j \in N$.

2) If $a_{i_{0} i_{0}} \neq 0$ and $a_{j_{0} j_{0}} \neq 0$ or $b_{i_{0} i_{0}} \neq 0$ and $b_{j_{0} j_{0}} \neq 0$ for some $i_{0}, j_{0}$, but $a_{i i} b_{i i}=0, \forall i \in N$, then

$$
\rho(A \circ B) \leq\left(\rho\left(J_{A}^{(2)}\right) \rho\left(J_{B}^{(2)}\right)\right)^{\frac{1}{2}} \max _{i \neq j}\left\{\left(a_{i i} a_{i j}\right)^{\frac{1}{2}},\left(b_{i i} b_{j j}\right)^{\frac{1}{2}}\right\} .
$$

3) If $a_{i i}=0$ and $b_{i i}=0, \forall i \in N$, then

$$
\rho(A \circ B) \leq\left(\rho\left(J_{A}^{\prime(2)}\right) \rho\left(J_{B}^{\prime(2)}\right)\right)^{\frac{1}{2}} .
$$

4) If $a_{i_{0} i_{0}} b_{i_{0} i_{0}} \neq 0$ and $a_{j_{0} j_{0}} b_{j_{0} j_{0}} \neq 0$ for some $i_{0}, j_{0}$, then the upper bound of $\rho(A \circ B)$ is the maximum value of the upper bounds of the inequalities in (9)-(11).

Proof. It is evident that 4) holds with equality for $n=1$. Next, we assume that $n \geq 2$.

(1) First, we assume that $A \circ B$ is irreducible matrix, then $A$ and $B$ are irreducible. Obviously $J_{A}^{\prime}$ and $J_{B}^{\prime}$ are also irreducible and nonnegative, so $J_{A}^{\prime(2)}$ and $J_{B}^{\prime(2)}$ are nonnegative irreducible matrices. Then there exist two positive vectors $\hat{u}=\left(\hat{u}_{i}\right)$ and $\hat{v}=\left(\hat{v}_{i}\right)$ such that $J_{A}^{\prime(2)} \hat{u}=\rho\left(J_{A}^{\prime(2)}\right) \hat{u}$ and $J_{B}^{\prime(k)} \hat{v}=\rho\left(J_{B}^{\prime(k)}\right) \hat{v}$. Let

$$
u=\left(u_{i}\right)=\hat{u}^{\left(\frac{1}{2}\right)}=\left(\hat{u}_{i}^{\frac{1}{2}}\right), \quad v=\left(v_{i}\right)=\hat{v}^{\left(\frac{1}{2}\right)}=\left(\hat{v}_{i}^{\frac{1}{2}}\right) .
$$

Then we have $J_{A}^{\prime(2)} u^{(2)}=\rho\left(J_{A}^{\prime(2)}\right) u^{(2)}$ and $J_{B}^{\prime(2)} v^{(2)}=\rho\left(J_{B}^{\prime(2)}\right) v^{(2)}$, that is

$$
\sum_{j \neq i} \frac{a_{i j}^{2} u_{j}^{2}}{u_{i}^{2}}=d_{i i}^{2} \rho\left(J_{A}^{\prime(2)}\right), \quad \sum_{j \neq i} \frac{b_{i j}^{2} v_{j}^{2}}{v_{i}^{2}}=\delta_{i i}^{2} \rho\left(J_{B}^{\prime(2)}\right) .
$$

Let $\hat{A}=\left(\hat{a}_{i j}\right)=U^{-1} A U$ and $\hat{B}=\left(\hat{b}_{i j}\right)=V^{-1} B V$ in which $U$ and $V$ are the nonsingular diagonal matrices $U=\operatorname{diag}\left(u_{1}, u_{2}, \cdots, u_{n}\right)$ and $V=\operatorname{diag}\left(v_{1}, v_{2}, \cdots, v_{n}\right)$. Then we have

$$
\begin{gathered}
\hat{A}=\left(\hat{a}_{i j}\right)=U^{-1} A U=\left(\begin{array}{cccc}
a_{11} & \frac{a_{12} u_{2}}{u_{1}} & \cdots & \frac{a_{1 n} u_{n}}{u_{1}} \\
\frac{a_{21} u_{1}}{u_{2}} & a_{22} & \cdots & \frac{a_{2 n} u_{n}}{u_{2}} \\
\vdots & \vdots & \ddots & \vdots \\
\frac{a_{n 1} u_{1}}{u_{n}} & \frac{a_{n 2} u_{2}}{u_{n}} & \cdots & a_{n n}
\end{array}\right), \\
\hat{B}=\left(\hat{b}_{i j}\right)=V^{-1} B V=\left(\begin{array}{cccc}
b_{11} & \frac{b_{12} v_{2}}{v_{1}} & \cdots & \frac{b_{1 n} v_{n}}{v_{1}} \\
\frac{b_{21} v_{1}}{v_{2}} & b_{22} & \cdots & \frac{b_{2 n} v_{n}}{v_{2}} \\
\vdots & \vdots & \ddots & \vdots \\
\frac{b_{n 1} v_{1}}{v_{n}} & \frac{b_{n 2} v_{2}}{v_{n}} & \cdots & b_{n n}
\end{array}\right) .
\end{gathered}
$$


It is easy to see that $\hat{A}, \hat{B}$, and $V U$ are nonsingular since $V$ and $U$ are. From Lemma 4, we have

$$
(V U)^{-1}(A \circ B)(V U)=U^{-1} V^{-1}(A \circ B) V U=\left(U^{-1} A U\right) \circ\left(V^{-1} B V\right)=\hat{A} \circ \hat{B} .
$$

Thus, we obtain $\rho(A \circ B)=\rho(\hat{A} \circ \hat{B})$, and

$$
\hat{A} \circ \hat{B}=\left(\hat{c}_{i j}\right)=\left(\begin{array}{cccc}
a_{11} b_{11} & \frac{a_{12} b_{12} u_{2} v_{2}}{u_{1} v_{1}} & \cdots & \frac{a_{1 n} b_{1 n} u_{n} v_{n}}{u_{1} v_{1}} \\
\frac{a_{21} b_{21} u_{1} v_{1}}{u_{2} v_{2}} & a_{22} b_{22} & \cdots & \frac{a_{2 n} b_{2 n} u_{n} v_{n}}{u_{2} v_{2}} \\
\vdots & \vdots & \ddots & \vdots \\
\frac{a_{n 1} b_{n 1} u_{1} v_{1}}{u_{n} v_{n}} & \frac{a_{n 2} b_{n 2} u_{2} v_{2}}{u_{n} v_{n}} & \cdots & a_{n n} b_{n n}
\end{array}\right) .
$$

We next consider the minimum eigenvalue $\rho(\hat{A} \circ \hat{B})$ of $\hat{A} \circ \hat{B}$. For nonnegative irreducible matrices $\hat{A}$ and $\hat{B}$, by definition of the Hadamard product of $\hat{A}$ and $\hat{B}$, Hölder's inequality, and Lemma 5, we have

$$
\begin{aligned}
& \rho(\hat{A} \circ \hat{B}) \leq \max _{i \neq j} \frac{1}{2}\left\{\hat{c}_{i i}+\hat{c}_{j j}+\left[\left(\hat{c}_{i i}-\hat{c}_{j j}\right)^{2}+4 \sum_{t \neq i} \hat{c}_{i t} \sum_{t \neq j} \hat{c}_{j t}\right]^{\frac{1}{2}}\right\} \\
& =\max _{i \neq j} \frac{1}{2}\left\{a_{i i} b_{i i}+a_{i j} b_{j j}+\left[\left(a_{i i} b_{i i}-a_{i j} b_{j j}\right)^{2}+4 \sum_{t \neq i} \frac{a_{i t} b_{i t} u_{t} v_{t}}{u_{i} v_{i}} \sum_{t \neq j} \frac{a_{j t} b_{j t} u_{t} v_{t}}{u_{j} v_{j}}\right]^{\frac{1}{2}}\right\} \\
& \leq \max _{i \neq j} \frac{1}{2}\left\{a_{i i} b_{i i}+a_{j j} b_{j j}+\left[\left(a_{i i} b_{i i}-a_{j j} b_{j j}\right)^{2}+4\left(\sum_{t \neq i} \frac{a_{i t}^{2} u_{t}^{2}}{u_{i}^{2}} \sum_{t \neq i} \frac{b_{i t}^{2} v_{t}^{2}}{v_{i}^{2}} \sum_{i \neq j} \frac{a_{j t}^{2} u_{t}^{2}}{u_{j}^{2}} \sum_{t \neq j} \frac{b_{j t}^{2} v_{t}^{2}}{v_{j}^{2}}\right)^{\frac{1}{2}}\right]^{\frac{1}{2}}\right\} \\
& =\max _{i \neq j} \frac{1}{2}\left\{a_{i i} b_{i i}+a_{i j} b_{j j}+\left[\left(a_{i i} b_{i i}-a_{i j} b_{j j}\right)^{2}+4 d_{i i} d_{i j} \delta_{i i} \delta_{j j} \rho\left(J_{A}^{(2)}\right) \rho\left(J_{B}^{(2)}\right)\right]^{\frac{1}{2}}\right\} .
\end{aligned}
$$

Thus, we obtain

1) If $a_{i i} b_{i i} \neq 0, \forall i \in N$, then

$$
\rho(A \circ B) \leq \max _{i \neq j} \frac{1}{2}\left\{a_{i i} b_{i i}+a_{j j} b_{j j}+\left[\left(a_{i i} b_{i i}-a_{i j} b_{j j}\right)^{2}+4 \eta_{i j}^{(2)}\right]^{\frac{1}{2}}\right\} .
$$

2) If $a_{i_{0} i_{0}} \neq 0$ and $a_{j_{0} j_{0}} \neq 0$ or $b_{i_{0} i_{0}} \neq 0$ and $b_{j_{0} j_{0}} \neq 0$ for some $i_{0}, j_{0}$, but $a_{i i} b_{i i}=0, \forall i \in N$, then

$$
\rho(A \circ B) \leq\left(\rho\left(J_{A}^{(2)}\right) \rho\left(J_{B}^{\prime(2)}\right)\right)^{\frac{1}{2}} \max _{i \neq j}\left\{\left(a_{i i} a_{i j}\right)^{\frac{1}{2}},\left(b_{i i} b_{j j}\right)^{\frac{1}{2}}\right\} .
$$

3) If $a_{i i}=0$ and $b_{i i}=0, \forall i \in N$, then

$$
\rho(A \circ B) \leq\left(\rho\left(J_{A}^{(2)}\right) \rho\left(J_{B}^{(2)}\right)\right)^{\frac{1}{2}} .
$$

4) If $a_{i_{0} i_{0}} b_{i_{0} i_{0}} \neq 0$ and $a_{j_{0} j_{0}} b_{j_{0} j_{0}} \neq 0$ for some $i_{0}, j_{0}$, then the upper bound of $\rho(A \circ B)$ is the maximum value of the upper bounds of the inequalities in (9)-(11). 
(2) Now, we assume that $A \circ B$ is reducible. If we denote by $T=\left(t_{i j}\right)$ the $n \times n$ permutation matrix with $t_{12}=t_{23}=\cdots=t_{n-1, n}=t_{n 1}=1$, the remaining $t_{i j}=0$, then both $A+\epsilon T$ and $B+\epsilon T$ are irreducible nonsingular matrices for any chosen positive real number $\epsilon$. Now, we substitute $A+\epsilon T$ and $B+\epsilon T$ for $A$ and $B$, respectively, in the previous case, and then letting $\epsilon \rightarrow 0$, the result follows by continuity.

Remark 2. By Lemma 2, the bound in Theorem 2 is better than that in Theorem 6 of [6] and Theorem 3 of [9]. Example 2. Let

$$
A=\left(\begin{array}{cccc}
2 & 0 & 1 & 1 \\
1 & 4 & 0.5 & 0.5 \\
1 & 0 & 3 & 0.5 \\
0.5 & 1 & 1 & 2
\end{array}\right), \quad B=\left(\begin{array}{cccc}
2 & 0.5 & 0.5 & 0.5 \\
1 & 1 & 1 & 1 \\
0.5 & 0 & 2 & 0.5 \\
0 & 1 & 1 & 2
\end{array}\right) .
$$

By calculation with Matlab 7.1, we have $\rho\left(J_{A}^{\prime}\right)=0.8182, \rho\left(J_{B}^{\prime}\right)=1.1258, \rho\left(J_{A}^{\prime(2)}\right)=0.3047$, $\rho\left(J_{B}^{\prime(2)}\right)=0.6263$, and $\rho(A \circ B)=6.3365$.

If we apply Theorem 6 of [4], Theorem 3 of [7], and Theorem 2.2 of [9], we have $\rho(A \circ B) \leq 11.5266$, $\rho(A \circ B) \leq 9.6221$, and $\rho(A \circ B) \leq 9.4116$, respectively. But, if we apply Theorem 2 , we have

$$
\rho(A \circ B) \leq \max _{i \neq j} \frac{1}{2}\left\{a_{i i} b_{i i}+a_{j j} b_{j j}+\left[\left(a_{i i} b_{i i}-a_{i j} b_{j j}\right)^{2}+4 \eta_{i j}^{(2)}\right]^{\frac{1}{2}}\right\}=7.3620 .
$$

The numerical example shows that the bound in Theorem 2 is better than that in Theorem 6 of [4], Theorem 3 of [7], and Theorem 2.2 of [9].

\section{References}

[1] Berman, A. and Plemmons, R.J. (1979) Nonnegaive Matrices in the Mathematical Sciences. Academic Press, New York.

[2] Horn, R.A. and Johnson, C.R. (1985) Topics in Matrix Analysis. Cambridge University Press, Cambridge. http://dx.doi.org/10.1017/CBO9780511810817

[3] Fang, M.Z. (2007) Bounds on Eigenvalues for the Hadamard Product and the Fan Product of Matrices. Linear Algebra and its Applications, 425, 7-15. http://dx.doi.org/10.1016/j.laa.2007.03.024

[4] Huang, R. (2008) Some Inequalities for the Hadamard Product and the Fan Product of Matrices. Linear Algebra and its Applications, 428, 1551-1559. http://dx.doi.org/10.1016/j.laa.2007.10.001

[5] Li, Y.T., Li, Y.Y., Wang, R.W. and Wang, Y.Q. (2010) Some New Lower Bounds on Eigenvalues of the Hadamard Product and the Fan Product of Matrices. Linear Algebra and its Applications, 432, 536-545. http://dx.doi.org/10.1016/j.laa.2009.08.036

[6] Liu, Q.B. and Chen, G.L. (2009) On Two Inequalities for the Hadamard Product and the Fan Product of Matrices. Linear Algebra and its Applications, 431, 974-984. http://dx.doi.org/10.1016/j.laa.2009.03.049

[7] Liu, Q.B., Chen, G.L. and Zhao, L.L. (2010) Some New Bounds on the Spectral Radius of Matrices. Linear Algebra and its Applications, 432, 936-948. http://dx.doi.org/10.1016/j.laa.2009.10.006

[8] Zhou, D.M., Chen, G.L., Wu, G.X. and Zhang, X.Y. (2013) On Some New Bounds for Eigenvalues of the Hadamard Product and the Fan Product of Matrices. Linear Algebra and its Applications, 438, 1415-1426. http://dx.doi.org/10.1016/j.laa.2012.09.013

[9] Zhao, L.L. (2012) Two Inequalities for the Hadamard Product of Matrices. Journal of Inequalities and Applications, 2012, 1-7. http://dx.doi.org/10.1186/1029-242X-2012-122

[10] Varga, R.S. (1962) Matrix Iterative Analysis. Prentice-Hall, Englewood Cliffs.

[11] Berman, A. and Plemmons, R.J. (1994) Nonnegaive Matrices in the Mathematical Sciences. SIAM, Philadelphia. http://dx.doi.org/10.1137/1.9781611971262

[12] Brauer, A. (1947) Limits for the Characteristic Roots of a Matrix II . Duke Mathematical Journal, 14, 21-26. http://dx.doi.org/10.1215/S0012-7094-47-01403-8 